\title{
Relación entre mortalidad y tratamiento recibido en pacientes con endocarditis infecciosa
}

\author{
Relationship between mortality and treatment received in patients with infective \\ endocarditis
}

\author{
Juan E. Gómez-Mesa ${ }^{1 *}$, Camilo Calderón-Miranda², Camilo Romo-Escorcia², Stephania Galindo-Coral', \\ Angy Cruz-Suárez ${ }^{3}$ y Pablo Moncada-Vallejo ${ }^{4}$ \\ ${ }^{1}$ Servicio de Cardiología y Falla Cardíaca, Fundación Valle del Lili; ${ }^{2}$ Departamento de Medicina Interna, Fundación Valle del Lili; ${ }^{3}$ Departamento de \\ Epidemiología, Universidad del Valle; ${ }^{4}$ Servicio de Infectología, Fundación Valle del Lili. Cali, Colombia
}

\section{Resumen}

Introducción: La endocarditis infecciosa es una enfermedad de baja incidencia y alta morbilidad. Puede tener un curso agudo o subagudo, con complicaciones que están directamente relacionadas con la mortalidad y varían según el agente infeccioso; el más frecuente es Staphylococcus aureus. Dependiendo de la condición clínica se puede elegir una terapia antimicrobiana exclusiva o una terapia combinada (antimicrobiana y cirugía); la elección adecuada impacta en las tasas de sobrevida. Objetivo: Describir las características clínicas, microbiológicas y ecocardiográficas en pacientes con endocarditis infecciosa y analizar la mortalidad según el tratamiento recibido (terapia antimicrobiana o terapia combinada). Método: Estudio de cohorte retrospectivo, realizado en el Hospital Universitario Fundación Valle del Lili, en Cali, Colombia, en el que se incluyeron pacientes hospitalizados mayores de 18 años que cumplían los criterios de Duke modificados para diagnóstico de endocarditis infecciosa. Se evaluaron dos cohortes según hubieran recibido tratamiento antimicrobiano exclusivo o terapia combinada. Los pacientes fueron seguidos hasta el egreso hospitalario. Resultados: De 101 pacientes, 58 recibieron terapia antimicrobiana y 43 terapia combinada. La sobrevida global fue del 77.35\%; el grupo de terapia combinada tuvo una hazard ratio ajustada de 0.13 (intervalo de confianza del 95\%: 0.036-0.505; $p=0.003$ ). La tasa de mortalidad calculada por 1000 días-persona fue de 25.9 con terapia antimicrobiana y de 4.33 con terapia combinada. Conclusiones: El grupo de terapia combinada tuvo más complicaciones y mayor estancia en la unidad de cuidados intensivos, pero menores tasas de mortalidad que los pacientes con terapia antimicrobiana exclusiva. De acuerdo con las indicaciones actuales de manejo quirúrgico descritas en las guías internacionales, se pueden obtener excelentes resultados en los pacientes más enfermos.

Palabras clave: Endocarditis. Mortalidad. Cirugía. Falla cardiaca.

\section{Abstract}

Introduction: Infective endocarditis is a disease of low incidence but high morbidity. It can have an acute or subacute course, with complications that are directly related to mortality and vary according to the infectious agent; the most common is Staphylococcus aureus. Based on the clinical condition, an exclusive antimicrobial therapy or a combined therapy (antimicrobial

\section{Correspondencia:}

*Juan E. Gómez-Mesa

E-mail: juan.gomez.me@fvl.org.co
Disponible en internet: $29-07-2021$

Rev Colomb Cardiol. 2021;28(3):246-253

www.rccardiologia.com 0120-5633 / @ 2020 Sociedad Colombiana de Cardiología y Cirugía Cardiovascular. Publicado por Permanyer. Este es un artículo open access bajo la licencia CC BY-NC-ND (http://creativecommons.org/licenses/by-nc-nd/4.0/). 
and surgery) can be chosen; their proper choice impacts survival rates. Objective: To describe the clinical, microbiological and echocardiographic characteristics in patients with infective endocarditis and to analyze how mortality behaved according to the treatment received (antimicrobial therapy or combined therapy). Method: A retrospective cohort study, carried out at the Fundación Valle del Lili University Hospital, Cali, Colombia, which included hospitalized patients over 18 years of age who met modified Duke criteria for the diagnosis of infective endocarditis. Two cohorts were evaluated according to treatment: exclusive antimicrobial and combined therapy. They were followed until discharge from the hospital. Results: Of 101 patients, 58 received antimicrobial therapy and 43 combined therapy. Overall survival was $77.35 \%$, the combined therapy group had an adjusted hazard ratio of 0.13 (95\% confidence interval: $0.036-0.505 ; p=0.003$ ). The mortality rate calculated per 1000 person-days was 25.9 in antimicrobial therapy and 4.33 in combination therapy. Conclusions: the combined therapy group had more complications and a longer hospital stay in the ICU, but lower mortality rates than the patients on exclusive antimicrobial therapy. According to the current indications for surgical management described in international guidelines, excellent results can be obtained in sicker patients.

Key words: Endocarditis. Mortality. Surgery. Heart failure.

\section{Introducción}

La endocarditis infecciosa tiene una incidencia que va de 1.5 hasta 9.6 casos por 100,000 habitantes ${ }^{1-3}$, y se asocia con elevadas morbilidad y mortalidad durante su curso. En Colombia hay pocas publicaciones referentes a datos epidemiológicos y la evidencia está dada por pequeños registros y series de $\operatorname{casos}^{4,5}$.

Por otra parte, el riesgo de endocarditis infecciosa está asociado con cambios estructurales causados por valvulopatía degenerativa o reumática, o por implantación de válvulas protésicas mecánicas y biológicas, catéteres venosos centrales o dispositivos cardioimplantables ${ }^{6-8}$, entre otros. La condiciones de comorbilidad que aumentan el riesgo de presentar endocarditis infecciosa incluyen diabetes mellitus (odds ratio [OR]: 3.5; intervalo de confianza del 95\% [IC95\%]: 1.03-12.97), insuficiencia cardiaca (OR: 3.12; IC95\%: 1.13-8.69) ${ }^{7}$, infección por el virus de la inmunodeficiencia humana (VIH), enfermedad renal crónica, enfermedad pulmonar obstructiva crónica y uso de drogas intravenosas ${ }^{7,9,10}$. Staphylococcus aureus es el agente etiológico más común y se asocia con mayor mortalidad, mientras que otros patógenos menos frecuentes son Streptococcus viridans, Staphylococcus coagulasa negativos, Enterococcus spp., Streptococcus bovis, Pseudomonas aeruginosa y hongos tipo Candida albicans ${ }^{11,12}$.

Las guías internacionales recomiendan cirugía (para control de la infección mediante eliminación de material infectado y drenaje de abscesos) en pacientes con endocarditis infecciosa e insuficiencia cardiaca grave, o si hay lesiones obstructivas que causan síntomas (clase I, nivel B), infección incontrolada por complicaciones locales como abscesos, pseudoaneurismas 0 fístulas (clase I, nivel B), si no se controla la bacteriemia a pesar de tratamiento antimicrobiano dirigido (clase lla, nivel B), o si las vegetaciones aórticas o mitrales miden más de $10 \mathrm{~mm}$ (clase I, nivel B). Existen otras indicaciones, pero con menor nivel de evidencia $^{13}$.

Los pacientes con endocarditis infecciosa de válvula nativa que cumplen criterios quirúrgicos y son llevados a cirugía temprana tienen una baja tasa de mortalidad intrahospitalaria (OR: 0.57 ; IC95\%: 0.42-0.77; $p=0.000$ ) y a largo plazo (OR: 0.57; IC95\%: 0.43-0.77; $p=0.001$ ), mientras que los pacientes con endocarditis infecciosa de válvula protésica no presentan diferencias significativas en mortalidad hospitalaria entre cirugía temprana

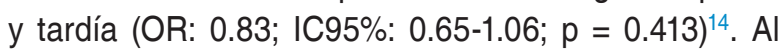
realizar una comparación entre la terapia antimicrobiana sola y la terapia combinada, en un estudio que incluyó 203 pacientes con endocarditis infecciosa, se encontró una mortalidad del $22 \%$ si eran llevados a cirugía y de un $32 \%$ si se elegía manejo antibiótico solo ${ }^{15}$. En otro estudio con pacientes con endocarditis infecciosa posterior a reemplazo valvular aórtico transcatéter se encontró que la mortalidad a 1 año no presentaba una diferencia estadísticamente significativa entre los dos grupos de intervenciones (68.2 vs. $65 \% ; p=0.82)^{16}$. La insuficiencia renal, la edad mayor de 77 años y la endocarditis infecciosa por $S$. aureus se han asociado con mayor mortalidad hospitalaria, a la vez que la insuficiencia renal y la cirugía tardía se han considerado predictores de mortalidad hospitalaria ${ }^{17}$.

El presente estudio tiene como objetivo describir las características clínicas, microbiológicas y ecocardiográficas de pacientes con endocarditis infecciosa, y analizar cómo se comporta la mortalidad según el tratamiento, ya sea con terapia antimicrobiana exclusiva o con terapia antimicrobiana y cirugía complementaria. 


\section{Método}

Se realizó un estudio de cohortes, retrospectivo, en el que se incluyeron pacientes hospitalizados entre enero de 2011 y junio de 2017, con diagnóstico de endocarditis infecciosa, en el Hospital Universitario Fundación Valle del Lili.

El protocolo fue revisado y aprobado por el Comité de Ética institucional, que provee una excepción a la realización de consentimiento informado. Por medio del sistema de diligenciamiento institucional (SAP), se identificaron los pacientes con diagnóstico de endocarditis infecciosa (códigos CIE-10: B376, I011, I33, I330, I339, I38, I38X, I39, I398) admitidos en la institución en el periodo de tiempo determinado.

Se incluyeron pacientes mayores de 18 años que cumplieran los criterios de Duke modificados para endocarditis infecciosa definida según las guías de práctica clínica de la Sociedad Europea de Cardiología de 2015. Se excluyeron los pacientes cuyo diagnóstico hubiera sido realizado en otra institución, no contaran con resultados de hemocultivos o de estudios histopatológicos, o que hubieran sido intervenidos de cirugía valvular o de retiro de dispositivo previamente al ingreso hospitalario. Los pacientes recibieron cirugía y terapia antimicrobiana (combinado) si tenían insuficiencia valvular (aórtica o mitral) aguda grave, obstrucción o fistula con edema pulmonar refractario, choque cardiogénico, infección localmente incontrolada (por absceso, pseudoaneurisma, fistula o vegetación $>15 \mathrm{~mm}$, insuficiencia valvular grave o embolia séptica), infección por hongos o hemocultivos persistentemente positivos a pesar del tratamiento antibiótico dirigido, y finalmente si el grupo tratante consideraba esta terapia. Los pacientes que no cumplían estas condiciones recibieron terapia antimicrobiana establecida por el servicio de infectología teniendo en cuenta el aislamiento de patógenos.

Se consideraron las características basales de cada grupo de pacientes según la estrategia de tratamiento, incluyendo variables demográficas, como edad y sexo, comorbilidad, características clínicas, ecocardiográficas y aislamientos microbiológicos más frecuentes. Se describieron y analizaron las variables relacionadas con complicaciones propias de la enfermedad, tiempo de estancia en la unidad de cuidados intensivos (UCl) y mortalidad.

Se incluyeron todos los pacientes diagnosticados de la enfermedad y que tuvieran registro en su historia clínica según los códigos $\mathrm{CIE}-10$ relacionados.
Se realizó un análisis descriptivo de las variables. En el análisis univariado se utilizaron la prueba de ji al cuadrado y la prueba exacta de Fisher para determinar diferencias entre los grupos de estrategias terapéuticas respecto a las características demográficas y microbiológicas, las manifestaciones clínicas y la comorbilidad. Para comparar las variables continuas se utilizó la prueba de Wilcoxon. El análisis de sobrevida se hizo con el método de Kaplan-Meier. Las diferencias en la sobrevida entre los grupos fueron evaluadas mediante log-rank test. Un valor de $p<0.05$ fue considerado significativo, y para todos los análisis estadísticos se presentan los IC95\%. Todos los análisis se realizaron con STATA® versión 15.0 (StataCorp LP, College Station, TX, USA). La sobrevida general se definió como el tiempo transcurrido entre la fecha del diagnóstico de endocarditis infecciosa y la fecha de la muerte o del último seguimiento registrado. Para determinar la diferencia en cuanto a sobrevida entre ambas estrategias de tratamiento observadas y ajustadas por confusores se utilizó un modelo de riesgos proporcionales de Cox.

\section{Resultados}

Entre enero de 2011 y junio de 2017 se diagnosticaron 101 casos de endocarditis infecciosa, con predominio del sexo masculino (67\%) y una mediana de edad de 60 años (rango: 47-69). La comorbilidad más frecuente incluyó insuficiencia cardiaca (31.68\%), enfermedad renal crónica (61.3\%), diabetes mellitus tipo $2(22.77 \%)$ y valvulopatías, entre las que se encontraron estenosis aórtica $(23.76 \%$ ) y mitral $(4.95 \%)$, e insuficiencia mitral $(28.71 \%)$, tricuspídea $(16.83 \%)$ y aórtica (11.88\%). El $65.3 \%$ de los pacientes tenían reemplazo valvular previo, mientras que el uso de esteroides y antibióticos durante los 3 meses anteriores al diagnóstico fue del $13.86 \%$ y el $46.53 \%$, respectivamente. La endocarditis infecciosa asociada a dispositivos ocurrió en el $86.14 \%$, incluyendo catéter implantable (25.74\%), cardiodesfibrilador implantable (6.93\%), marcapasos transcutáneo (7.92\%) y catéter venoso central (86.14\%) (Tabla 1).

La fiebre fue la manifestación clínica más frecuente $(84.16 \%)$, seguida de fenómenos vasculares aislados (13.86\%). Las válvulas con mayor compromiso fueron la mitral $(33.66 \%)$ y la aórtica $(29.7 \%)$. El ecocardiograma mostró vegetación $(79.2 \%)$ y perforación valvular $(8.91 \%)$. Los patógenos predominantes fueron S. aureus (31.68\%) y Streptococcus spp. (13.86\%); se obtuvieron cultivos positivos independientemente del patógeno en cultivo de vegetación (70.3\%), punta de catéter (69.31\%) 
Tabla 1. Características generales de los pacientes con endocarditis infecciosa por grupo de terapia

\begin{tabular}{|c|c|c|c|c|c|c|c|}
\hline & \multicolumn{2}{|c|}{$\begin{array}{c}\text { Total } \\
(\mathrm{n}=101)\end{array}$} & \multicolumn{2}{|c|}{$\begin{array}{l}\text { Antimicrobiana } \\
\qquad(\mathrm{n}=58)\end{array}$} & \multicolumn{2}{|c|}{$\begin{array}{l}\text { Combinada } \\
(\mathrm{n}=43)\end{array}$} & \multirow[b]{2}{*}{$\mathbf{p}$} \\
\hline & n & $\%$ & n & $\%$ & n & $\%$ & \\
\hline Edad, mediana (rango) & \multicolumn{2}{|c|}{$60(47-69)$} & \multicolumn{2}{|c|}{$64(50-75)$} & \multicolumn{2}{|c|}{$56(37-62)$} & $0.0211^{*}$ \\
\hline $\begin{array}{l}\text { Sexo } \\
\text { Masculino }\end{array}$ & 67 & 66.34 & 41 & 70.69 & 26 & 60.47 & $0.282^{\dagger}$ \\
\hline $\begin{array}{l}\text { Antecedentes } \\
\text { Enfermedad periodontal } \\
\text { Falla cardiaca }\end{array}$ & $\begin{array}{l}56 \\
32\end{array}$ & $\begin{array}{l}55.45 \\
31.68\end{array}$ & $\begin{array}{l}31 \\
18\end{array}$ & $\begin{array}{l}53.45 \\
31.03\end{array}$ & $\begin{array}{l}25 \\
14\end{array}$ & $\begin{array}{l}58.14 \\
32.56\end{array}$ & $\begin{array}{l}0.908^{\ddagger} \\
0.871^{\dagger}\end{array}$ \\
\hline $\begin{array}{l}\text { Estenosis valvular } \\
\text { Mitral } \\
\text { Tricuspídea } \\
\text { Aórtica }\end{array}$ & $\begin{array}{c}5 \\
1 \\
24\end{array}$ & $\begin{array}{c}4.95 \\
0.99 \\
23.76\end{array}$ & $\begin{array}{c}3 \\
0 \\
13\end{array}$ & $\begin{array}{c}5.17 \\
0.00 \\
22.41\end{array}$ & $\begin{array}{c}2 \\
1 \\
11\end{array}$ & $\begin{array}{c}4.65 \\
2.33 \\
25.58\end{array}$ & NA \\
\hline $\begin{array}{l}\text { Insuficiencia valvular } \\
\text { Mitral } \\
\text { Tricuspídea } \\
\text { Aórtica }\end{array}$ & $\begin{array}{l}29 \\
17 \\
12\end{array}$ & $\begin{array}{l}28.71 \\
16.83 \\
11.88\end{array}$ & $\begin{array}{l}14 \\
12 \\
7\end{array}$ & $\begin{array}{l}24.14 \\
20.69 \\
12.07\end{array}$ & $\begin{array}{l}15 \\
5 \\
5\end{array}$ & $\begin{array}{l}34.88 \\
11.63 \\
11.63\end{array}$ & NA \\
\hline Reemplazo valvular & 66 & 65.35 & 36 & 62.07 & 30 & 69.77 & $0.421^{\dagger}$ \\
\hline Endocarditis infecciosa previa & 8 & 7.92 & 4 & 6.90 & 4 & 9.30 & $0.720^{\ddagger}$ \\
\hline $\begin{array}{l}\text { Enfermedad renal crónica } \\
\text { Hemodiálisis }\end{array}$ & 61 & 60.40 & 36 & 62.07 & 25 & 58.14 & $0.817^{\ddagger}$ \\
\hline $\begin{array}{l}\text { Neoplasia } \\
\text { Sin neoplasia } \\
\text { Hematolinfoide } \\
\text { Órgano sólido }\end{array}$ & $\begin{array}{c}85 \\
9 \\
6\end{array}$ & $\begin{array}{c}84.16 \\
8.91 \\
6.93\end{array}$ & $\begin{array}{c}49 \\
5 \\
4\end{array}$ & $\begin{array}{c}84.48 \\
8.80 \\
7\end{array}$ & $\begin{array}{c}36 \\
4 \\
2\end{array}$ & $\begin{array}{c}83.72 \\
9.10 \\
4.50\end{array}$ & $0.825^{\ddagger}$ \\
\hline Diabetes mellitus tipo 2 & 23 & 22.77 & 13 & 22.41 & 10 & 23.26 & $0.921^{\dagger}$ \\
\hline $\begin{array}{l}\text { Dispositivos } \\
\text { Catéter implantable } \\
\text { Cardiodesfibrilador } \\
\text { implantable } \\
\text { Marcapasos } \\
\text { Catéter central }\end{array}$ & $\begin{array}{c}26 \\
\\
7 \\
8 \\
87\end{array}$ & $\begin{array}{c}25.74 \\
\\
6.93 \\
7.92 \\
86.14\end{array}$ & $\begin{array}{c}17 \\
\\
3 \\
5 \\
49\end{array}$ & $\begin{array}{c}29.31 \\
\\
5.17 \\
8.62 \\
84.48\end{array}$ & $\begin{array}{c}9 \\
\\
4 \\
3 \\
38\end{array}$ & $\begin{array}{c}20.93 \\
\\
9.30 \\
6.98 \\
88.37\end{array}$ & $\begin{array}{l}0.341^{\dagger} \\
\\
0.455^{\ddagger} \\
1.000^{\ddagger} \\
0.772^{\ddagger}\end{array}$ \\
\hline Uso de esteroides $<90$ días & 14 & 13.86 & 9 & 15.52 & 5 & 11.63 & $0.576^{\dagger}$ \\
\hline Uso de antibiótico $<90$ días & 47 & 46.53 & 23 & 39.66 & 24 & 55.81 & $0.107^{\dagger}$ \\
\hline
\end{tabular}

NA: no aplica.

*Prueba de Wilcoxon.

${ }^{\dagger}$ Prueba de ji al cuadrado.

${ }^{\ddagger}$ Prueba exacta de Fisher.

y hemocultivo (36.63\%). Las complicaciones más frecuentes asociadas a la endocarditis infecciosa fueron embolia pulmonar (68.32\%), embolia cerebral (65.35\%), rotura valvular $(61.39 \%)$, insuficiencia cardiaca aguda (47.52\%) y sepsis (40.59\%). Un $42.57 \%$ de los pacientes recibieron tratamiento combinado y a un $18.81 \%$ se les retiró el dispositivo (Tabla 2).

Los pacientes se dividieron en dos grupos: 58 con terapia antimicrobiana y 43 con terapia combinada (antimicrobiana y cirugía). Los grupos tuvieron un tiempo de seguimiento promedio de 22 y 44 días, respectivamente. La sobrevida global fue cercana al $77.35 \%$, pero la terapia combinada presentó mejor sobrevida a lo largo del periodo de observación, siendo más notoria la diferencia en los primeros 30 días (Fig. 1). No se observaron diferencias significativas respecto al tiempo para el diagnóstico entre ambos grupos. Los pacientes con terapia combinada tuvieron una estancia en la UCI más prolongada. La tasa de mortalidad calculada por 1000 días-persona fue de 25.9 para la terapia antimicrobiana y de 4.33 para la terapia combinada; cifras que indican que la tasa de mortalidad es 
Tabla 2. Hallazgos clínicos y paraclínicos de pacientes con endocarditis infecciosa por grupo de terapia

\begin{tabular}{|c|c|c|c|c|c|c|c|}
\hline & \multicolumn{2}{|c|}{$\begin{array}{c}\text { Total } \\
(\mathrm{n}=101)\end{array}$} & \multicolumn{2}{|c|}{$\begin{array}{l}\text { Antimicrobiana } \\
\quad(\mathrm{n}=\mathbf{5 8})\end{array}$} & \multicolumn{2}{|c|}{$\begin{array}{l}\text { Combinada } \\
(\mathrm{n}=43)\end{array}$} & \multirow[b]{2}{*}{$\mathbf{p}$} \\
\hline & n & $\%$ & n & $\%$ & n & $\%$ & \\
\hline $\begin{array}{l}\text { Presentación clínica } \\
\text { Fiebre } \\
\text { Fenómenos vasculares e inmunitarios }\end{array}$ & $\begin{array}{l}85 \\
25\end{array}$ & $\begin{array}{l}84.16 \\
24.75\end{array}$ & $\begin{array}{l}52 \\
16\end{array}$ & $\begin{array}{l}89.66 \\
27.59\end{array}$ & $\begin{array}{c}33 \\
9\end{array}$ & $\begin{array}{l}76.74 \\
20.93\end{array}$ & $\begin{array}{l}0.079^{*} \\
0.031^{\dagger}\end{array}$ \\
\hline $\begin{array}{l}\text { Aislamiento } \\
\text { Sin aislamiento } \\
\text { Staphylococcus aureus } \\
\text { Staphylococcus coagulasa negativos } \\
\text { Streptococcus spp. } \\
\text { Enterococcus } \\
\text { Gramnegativos } \\
\text { Hongos }\end{array}$ & $\begin{array}{c}16 \\
32 \\
10 \\
14 \\
11 \\
9 \\
5\end{array}$ & $\begin{array}{c}15.84 \\
31.68 \\
9.90 \\
13.86 \\
10.89 \\
8.91 \\
4.95\end{array}$ & $\begin{array}{c}7 \\
20 \\
6 \\
7 \\
6 \\
8 \\
2\end{array}$ & $\begin{array}{c}12.07 \\
34.48 \\
10.34 \\
12.07 \\
10.34 \\
13.79 \\
3.45\end{array}$ & $\begin{array}{c}9 \\
12 \\
4 \\
7 \\
5 \\
1 \\
3\end{array}$ & $\begin{array}{c}20.93 \\
27.91 \\
9.30 \\
16.28 \\
11.63 \\
2.33 \\
6.98\end{array}$ & $0.486^{\dagger}$ \\
\hline $\begin{array}{l}\text { Tipo de cultivo } \\
\text { Hemocultivo } \\
\text { Cultivo de punta de catéter } \\
\text { Cultivo de vegetación } \\
\text { Histopatología }\end{array}$ & $\begin{array}{l}37 \\
70\end{array}$ & $\begin{array}{l}36.63 \\
69.31\end{array}$ & $\begin{array}{l}22 \\
36\end{array}$ & $\begin{array}{l}37.93 \\
62.07\end{array}$ & $\begin{array}{l}15 \\
34 \\
31 \\
14\end{array}$ & $\begin{array}{c}34.88 \\
79.07 \\
72.09 \\
32.5\end{array}$ & $\begin{array}{c}0.753^{*} \\
0.067^{*} \\
\text { NA } \\
\text { NA }\end{array}$ \\
\hline \multicolumn{8}{|l|}{ Hallazgos en ecocardiograma } \\
\hline $\begin{array}{l}\text { Válvula afectada } \\
\text { Mitral } \\
\text { Aórtica } \\
\text { Tricuspídea }\end{array}$ & $\begin{array}{l}34 \\
30 \\
15\end{array}$ & $\begin{array}{l}33.66 \\
29.70 \\
14.85\end{array}$ & $\begin{array}{c}18 \\
16 \\
9\end{array}$ & $\begin{array}{l}31.03 \\
27.59 \\
15.52\end{array}$ & $\begin{array}{l}16 \\
14 \\
6\end{array}$ & $\begin{array}{l}37.21 \\
32.56 \\
13.95\end{array}$ & $0.812^{\dagger}$ \\
\hline $\begin{array}{l}\text { Complicaciones locales } \\
\text { Absceso perivalvular } \\
\text { Perforación valvular } \\
\text { Aneurisma valvular } \\
\text { Fístula insuficiencia cardiaca o } \\
\text { perivalvular } \\
\text { Engrosamiento de válvula } \\
\text { Rotura de cuerda } \\
\text { Ninguna }\end{array}$ & $\begin{array}{c}1 \\
9 \\
2 \\
72\end{array}$ & $\begin{array}{r}6.93 \\
8.91 \\
0.99 \\
\\
0.99 \\
8.91 \\
1.98 \\
71.29\end{array}$ & $\begin{array}{c}0 \\
3 \\
0 \\
48\end{array}$ & $\begin{array}{c}5.17 \\
82.76\end{array}$ & $\begin{array}{c}1 \\
6 \\
2 \\
24\end{array}$ & $\begin{array}{c}11.63 \\
9.30 \\
2.33 \\
\\
2.33 \\
13.95 \\
4.65 \\
55.81\end{array}$ & $0.027^{\dagger}$ \\
\hline $\begin{array}{l}\text { Endocarditis infecciosa dispositivo implantable con } \\
\text { vegetación }\end{array}$ & 58 & 57.43 & 34 & 58.62 & 24 & 55.81 & $0.778^{*}$ \\
\hline Endocarditis infecciosa válvula nativa & 51 & 50.50 & 32 & 55.17 & 19 & 44.19 & $0.485^{\dagger}$ \\
\hline No hallazgos ecocardiográficos & 12 & 11.88 & 7 & 12.07 & 5 & 11.63 & $0.560^{\dagger}$ \\
\hline $\begin{array}{l}\text { Complicaciones } \\
\text { Sepsis } \\
\text { Falla cardiaca aguda } \\
\text { Rotura valvular } \\
\text { Insuficiencia valvular } \\
\text { Embolia cerebral } \\
\text { Embolia pulmonar }\end{array}$ & $\begin{array}{l}41 \\
48 \\
62 \\
26 \\
66 \\
69\end{array}$ & $\begin{array}{l}40.59 \\
47.52 \\
61.39 \\
25.74 \\
65.35 \\
68.32\end{array}$ & $\begin{array}{l}22 \\
32 \\
38 \\
20 \\
36 \\
39\end{array}$ & $\begin{array}{l}37.93 \\
55.17 \\
65.52 \\
34.48 \\
62.07 \\
67.24\end{array}$ & $\begin{array}{c}19 \\
16 \\
24 \\
6 \\
30 \\
30\end{array}$ & $\begin{array}{l}44.19 \\
37.21 \\
55.81 \\
13.95 \\
69.77 \\
69.77\end{array}$ & $\begin{array}{l}0.527^{*} \\
0.070^{\dagger} \\
0.322^{*} \\
0.020^{*} \\
0.104^{\dagger} \\
0.267^{\dagger}\end{array}$ \\
\hline Retiro de dispositivo & 19 & 18.81 & 6 & 10.34 & 13 & 30.23 & $0.011^{*}$ \\
\hline
\end{tabular}

NA: no aplica.

*Prueba de ji al cuadrado.

tPrueba exacta de Fisher.

casi seis veces mayor en el grupo de terapia antimicrobiana (Tabla 3). La sobrevida con la terapia combinada de acuerdo con los hallazgos previos mostró una hazard ratio [HR] de 0.24 (IC95\%: 0.08-0,67; $p=0.007$ ), y al ajustarla por las variables de confusión, que fueron estadísticamente significativas (edad, antibiótico en los 90 días previos al diagnóstico, aislamiento en cultivo de punta de catéter, insuficiencia cardiaca aguda y retiro 
Tabla 3. Asociación observada y ajustada por confusores entre las dos estretegias de terapia y mortalidad

\begin{tabular}{|l|c|c|c|c|c|c|c|c|c|c|}
\hline Terapia & Casos & Tiempo* & \multirow{2}{*}{ Tasa $^{\dagger}$} & \multicolumn{3}{|c|}{ Observada } & \multicolumn{3}{|c|}{ Ajustada } \\
\cline { 5 - 11 } & & & & HR & IC95\% & p & HR & IC95\% & p \\
\hline Antimicrobiana & 17 & 657 & 25.9 & 1.00 & & & & & \\
\hline Combinada & 5 & 1154 & 4.33 & 0.24 & $0.08-0.67$ & 0.007 & 0.13 & $0.036-0.505$ & 0.003 \\
\hline
\end{tabular}

HR: hazard ratio; IC $95 \%$ : intervalo de confianza del $95 \%$.

*En días-persona.

†Tasa de mortalidad por 1000 días-persona.

Tabla 4. Asociación de confusores y mortalidad por endocarditis infecciosa $(\mathrm{n}=101)$

\begin{tabular}{|l|c|c|c|}
\hline & \multicolumn{3}{|c|}{ Ajustada } \\
\hline Variable & HR & IC95\% & p \\
\hline Edad & 1.035 & $1.000-1.071$ & 0.044 \\
\hline Antibiótico previo & 1.027 & $0.408-2.580$ & 0.954 \\
\hline Fiebre & 5.85 & $0.437-78.354$ & 0.182 \\
\hline Falla cardiaca aguda & 0.515 & $0.189-1.405$ & 0.195 \\
\hline Insuficiencia valvular & 0.428 & $0.116-1.573$ & 0.202 \\
\hline Cultivo de punta & 3.482 & $1.086-11.162$ & 0.036 \\
\hline Retiro de dispositivo & 2.221 & $0.602-8.191$ & 0.231 \\
\hline
\end{tabular}

HR: hazard ratio; IC95\%: intervalo de confianza del $95 \%$.

del dispositivo), se encontró una HR ajustada de 0.13 (IC95\%: 0.036-0.505; $p=0.003$ ) (Tablas 3-4).

\section{Discusión}

Aunque se han descrito numerosos síntomas y signos asociados a endocarditis infecciosa, los cuales son agrupados en los criterios de Duke modificados, estos no se presentan de forma constante, pero la fiebre sigue siendo la manifestación clínica más frecuen$t^{12,13}$. La fiebre y los fenómenos vasculares e inmunitarios se presentaron con más frecuencia en el grupo de terapia antimicrobiana, con una diferencia estadísticamente significativa. No se identificaron muchos pacientes con infección por VIH ni con uso previo de esteroides, lo cual puede influir en la poca frecuencia observada de infecciones por hongos, micobacterias y bacterias gramnegativas, que son comunes en los pacientes inmunosuprimidos ${ }^{18,19}$. Los patógenos más frecuentes siguen siendo $S$. aureus y Streptococcus spp. El uso de antibióticos en los 90 días previos al

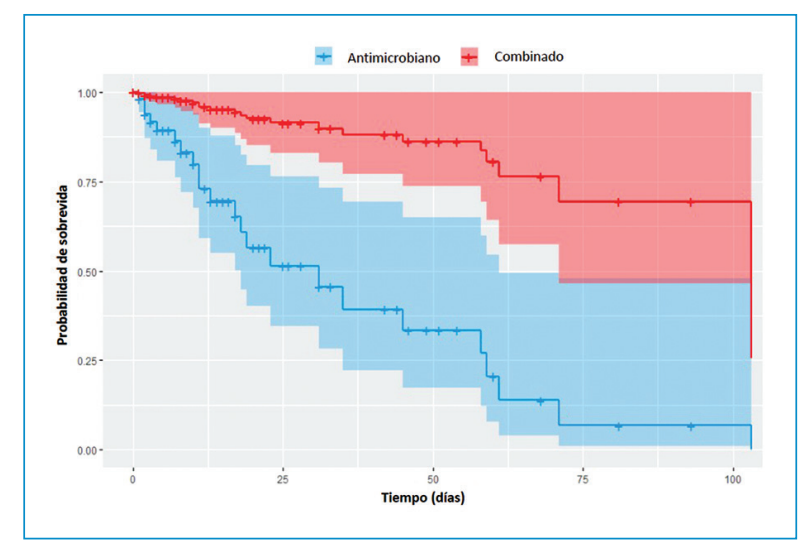

Figura 1. Curva de Kaplan-Meier para sobrevida relacionada con el tipo de terapia elegida en los pacientes con diagnóstico de endocarditis infecciosa.

diagnóstico es una de las principales causas de hemocultivos negativos, y en este estudio esto ocurrió en el $46.53 \%$. El cultivo de punta de catéter y el cultivo de la vegetación fueron las principales fuentes de aislamiento, y solo el $36.6 \%$ de los pacientes tuvieron hemocultivos positivos.

Se observó un mayor compromiso valvular izquierdo (mitral y aórtico); aunque los usuarios de drogas por vía intravenosa se presentan con mayor compromiso valvular derecho (tricuspídea y pulmonar), este factor de riesgo no se identificó en las historias clínicas evaluadas. El grupo de terapia combinada tuvo más complicaciones asociadas a endocarditis infecciosa, como absceso perivalvular, perforación valvular o rotura de cuerda tendinosa, así como más casos de insuficiencia cardiaca, insuficiencia valvular y embolias sistémicas, que condicionaron la elección de la terapia combinada ${ }^{12,13}$.

Se encontró que los pacientes con endocarditis infecciosa que recibieron terapia combinada eran más jóvenes, lo cual puede influir en el desenlace de sobrevida. No hubo diferencias estadísticamente significativas entre los dos grupos según los antecedentes, 
los factores de riesgo ni la comorbilidad previa al diagnóstico de endocarditis infecciosa. El grupo de terapia combinada tuvo mayor tiempo de seguimiento, lo cual es de esperar dado que estuvieron sometidos a intervención quirúrgica; por lo tanto, también tuvieron un mayor tiempo de estancia en la $\mathrm{UCl}$.

La tasa de mortalidad del grupo de terapia antimicrobiana comparada con la del grupo de terapia combinada es casi seis veces mayor. Aunque se podría pensar que los pacientes llevados a cirugía tienen mayores complicaciones y su condición clínica es más crítica, al analizar la asociación entre sobrevida y el uso de estrategia combinada la HR ajustada fue de 0.13 (IC95\%: 0.036-0.505; $p=0.003$ ), lo que no parece estar influenciado por las variables de confusión y las variables con diferencias estadísticas entre grupos, y no se está entregando el manejo quirúrgico a personas de peor pronóstico. Según estos datos, en esta institución, elegir la estrategia de terapia combinada (antimicrobiana y cirugía), siguiendo las recomendaciones de las guías internacionales, se asocia a una mejor sobrevida en comparación con los pacientes con terapia antimicrobiana sola, pero con un mayor tiempo de estancia en la $\mathrm{UCl}$.

Se requieren estudios adicionales, con un control más riguroso de los criterios, para decidir la indicación de manejo quirúrgico, evaluar las diferentes técnicas de intervención y estandarizar el tratamiento antimicrobiano, así como la inclusión de otros centros hospitalarios.

\section{Conclusiones}

A pesar de presentar un mayor tiempo de estancia en la UCl y más complicaciones, los pacientes que reciben terapia combinada (cirugía y antimicrobiano) tienen mejores tasas de sobrevida que aquellos en quienes se elige solamente terapia antimicrobiana, esto si se tiene en cuenta que deben seguirse de forma estricta las indicaciones de manejo quirúrgico descritas en las guías internacionales.

\section{Limitaciones}

En este estudio solo se incluyeron pacientes de una institución. Por otra parte, al ser un estudio observacional y basado en registros de pacientes, no es posible tener un estricto control de las variables. Adicionalmente, la cohorte dependía de la elección del tratamiento por el grupo tratante, mientras que la terapia antimicrobiana fue seleccionada por el servicio de infectología.

\section{Agradecimientos}

Los autores agradecen a Felipe Rosero y Juan David Agrado (estudiantes de medicina de la Universidad Icesi), a la Universidad CES de Medellín, al Centro de Investigaciones Clínicas y al Hospital Universitario Fundación Valle del Lili, de Cali.

\section{Financiamiento}

Este estudio no recibió ninguna ayuda económica específica de agencias de financiamiento en los sectores público, comercial o sin fines de lucro.

\section{Conflicto de intereses}

Los autores de este estudio no tienen ningún conflicto de intereses que declarar.

\section{Responsabilidades éticas}

Protección de personas y animales. Los autores declaran que para esta investigación no se han realizado experimentos en seres humanos ni en animales.

Confidencialidad de los datos. Los autores declaran que han seguido los protocolos de su centro de trabajo sobre la publicación de datos de pacientes.

Derecho a la privacidad y consentimiento informado. Los autores declaran que en este artículo no aparecen datos de pacientes.

\section{Bibliografía}

1. Ambrosioni J, Hernández-Meneses $M$, Téllez A, Pericàs J, Falces $C$, Tolosana JM, et al. Hospital Clinic Infective Endocarditis Investigators, 2017. The changing epidemiology of infective endocarditis in the twenty-first century. Curr Infect Dis Rep. 2017;19:21.

2. Cabell $\mathrm{CH}$, Heidenreich PA, Chu VH, Moore CM, Stryjewski ME, Corey GR, et al. Increasing rates of cardiac device infections among Medicare beneficiaries: 1990-1999. Am Heart J. 2004;147:582-6.

3. Cahill TJ, Baddour LM, Habib G, Hoen B, Salaun E, Pettersson GB, et al. Challenges in infective endocarditis. J Am Coll Cardiol. 2017;69:325-44.

4. Calvo N, Enrique I. Caracterización clínica, microbiológica y ecocardiográfica de pacientes con endocarditis infecciosa en la Fundación Cardioinfantil 2002-2012. (Tesis de Grado.) Bogotá: Universidad del Rosario; 2014.

5. Capilla E, Poyet R, Tortat AV, Marchi J, Brocq FX, Pons F, et al. Infective endocarditis: clinical features and prognosis between 2004 and 2014 in a non-teaching hospital. Ann Cardiol Angeiol (Paris). 2017;66:87-91.

6. Carrasco F, Anguita M, Ruiz M, Castillo JC, Delgado M, Mesa D, et al. Clinical features and changes in epidemiology of infective endocarditis on pacemaker devices over a 27-year period (1987-2013). Europace. 2016;18:836-41.

7. Gebo KA, Burkey MD, Lucas GM, Moore RD, Wilson LE. Incidence of, risk factors for, clinical presentation, and 1-year outcomes of infective endocarditis in an urban HIV cohort. J Acquir Immune Defic Syndr. 2006;43:426-32.

8. Habib G, Lancellotti $P$, Antunes MJ, Bongiorni MG, Casalta JP Del Zotti F, et al.; ESC Scientific Document Group, 2015. 2015 ESC Guidelines for the management of infective endocarditis: The Task Force for the Management of Infective Endocarditis of the European Society of Cardiology (ESC). Endorsed by: European Association for Cardio-Thoracic Surgery (EACTS), the European Association of Nuclear Medicine (EANM). Eur Heart J. 2015;36:3075-128. 
9. Hercé B, Nazeyrollas P, Lesaffre F, Sandras R, Chabert JP, Martin A, et al. Risk factors for infection of implantable cardiac devices: data from a registry of 2496 patients. Europace. 2013;15:66-70.

10. Krecki R, Drozdz J, Ibata G, Lipiec P, Ostrowski S, Kasprzak J, et al. Clinical profile, prognosis and treatment of patients with infective endocarditis - a 14-year follow-up study. Pol Arch Med. 2007;117: 512-20.

11. Liang F, Song B, Liu R, Yang L, Tang H, Li Y. Optimal timing for early surgery in infective endocarditis: a meta-analysis. Interact Cardiovasc Thorac Surg. 2016;22:336-45.

12. Mangner N, Leontyev S, Woitek FJ, Kiefer P, Haussig S, Binner C, et al. Cardiac surgery compared with antibiotics only in patients developing infective endocarditis after transcatheter aortic valve replacement. J Am Heart Assoc. 2018;7:e010027.

13. N'Guyen $Y$, Duval X, Revest M, Saada M, Erpelding ML, Selton-Suty C, et al.; AEPEI study group. Time interval between infective endocarditis first symptoms and diagnosis: relationship to infective endocarditis characteristics, microorganisms and prognosis. Ann Med. 2017; 49:117-25.
14. Nunes MCP, Guimarães-Júnior $\mathrm{MH}$, Murta Pinto PHO, Coelho RMP, Souza Barros TL, Faleiro Maia N, et al. Outcomes of infective endocarditis in the current era: early predictors of a poor prognosis. Int $\mathrm{J}$ Infect Dis. 2018;68:102-7.

15. Pant S, Patel NJ, Deshmukh A, Golwala H, Patel N, Badheka A, et al Trends in infective endocarditis incidence, microbiology, and valve replacement in the United States from 2000 to 2011. J Am Coll Cardiol. 2015;65:2070-6.

16. Rosso F, Cedano JA, Franco-Molina MA, Borrero-González AF, García-Robledo JE. Comparación de las características y curso clínico de la endocarditis infecciosa por Staphylococcus aureus meticilino sensible versus meticilino resistente. Rev Colomb Cardiol. 2018;25:314-20.

17. Tleyjeh IM, Abdel-Latif A, Rahbi H, Scott CG, Bailey KR, Steckelberg JM, et al. A systematic review of population-based studies of infective endocarditis. Chest. 2007;132:1025-35.

18. Vasudev R, Shah P, Bikkina M, Shamoon F. Infective endocarditis in HIV. Int J Cardiol. 2016;214:216-7.

19. Vogkou CT, Vlachogiannis NI, Palaiodimos L, Kousoulis AA. The causative agents in infective endocarditis: a systematic review comprising 33,214 cases. Eur J Clin Microbiol Infect Dis. 2016;35:1227-45. 